\title{
مستوى معرفة اتجاهات معلمي التعليم العام بطلبة صعوبات التعلم في ضوء بعض المتغيرات بدولة الكويت
}

\author{
أحمد محسن السعيدي \\ أستاذ مشارك في قسم التربية الخاصة _كلية التربية الأسـاسية \\ ahmed_alsadiey@yahoo.com
}

هدفت الدراسة إلى التعرف على مستوى معرفة اتجاهات معلمي التعليم العام بطلبة صعوبات التعلم في ضوء بعض المتغيرات بدولة الكويت كما هدفت أيضا إلى التعرف على الفروق بهذه الاتجاهات حسب متغير(الجنس، الخبرة التدريسية، المرحلة التعليمية) لدى(9rا7) من المعلمين

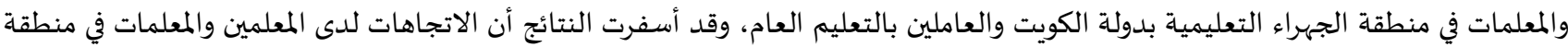

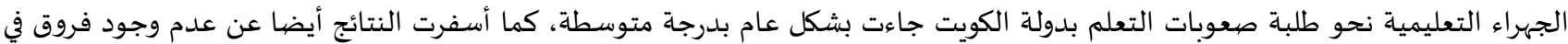
الاتجاهات لدى كل من المعلمين والمعلمات في منطقة الجهراء التعليمية تبعا لمتغير الجنس، والخبرة التدريسية ، والمرحلة التعليمية.

\section{(c) (1)}

الكلمات المفتاحية: الاتجاهات- صعوبات التعلم

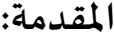

تعد صعوبات التعلم من الموضوعات المهمة في الوقت الراهن في مجال التربية الخاصة، والتي أعطيت اهتماما كبيرا من المهتمين علي اختلاف اختصاصاتهم كالأطباء وعلماء النفس وعلماء التربية والمعلمين وأولياء الأمور وغيرهم ، لتزايد أعدادها وبشكل رئيسي نتيجة ، للتطور الحاصل في عمليات

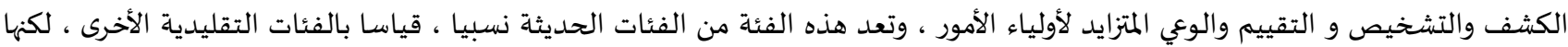

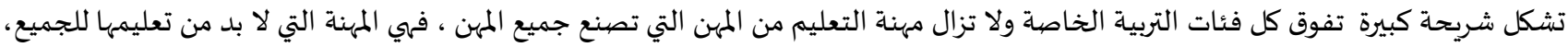

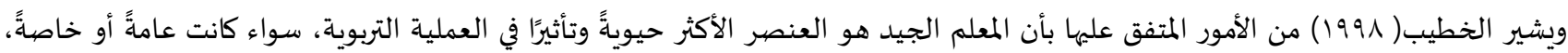

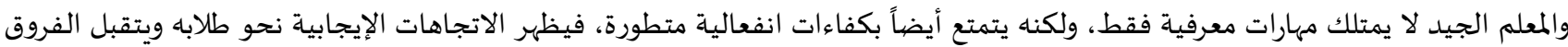

بينهم ويراعيها.

وتحتل الاتجاهات مكانا هاما في تحديد سلوك المعلمين تجاه طلابهم بصورة عامة ، واتجاههم نحو الطلاب من ذوي صعوبات التعلم بصورة خاصة فهي التي تؤثر في إدراكهم للمواقف والأحداث التي تربطهم معهم ، وفي حكمهه علي تلك المواقف التي يظهرها هؤلاء الطلبة ، كما أنها تؤثر في دوي الكفاءة والدافعية للعمل مع طلابهم من ذوي صعوبات التعلم

مشـكلة الدارسـة:

يتفق الجميع على أهمية الاتجاهات في حياة الإنسان بشكل عام وفي العملية التعليمية بوجه خاص فهي تعتبر نوع من الاستعد اد المسبق للفرد

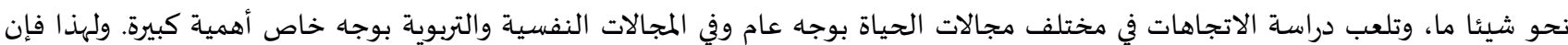
موضوع الاتجاهات نحو طلبة صعوبات التعلم من الموضوعات المهمة في ميدان الأفراد ذوي الاحتياجات الخاصة إذ يعود ذلك إلى عدد من الأسباب

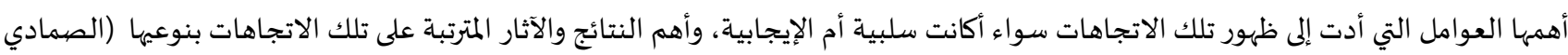
ومعابرة، 7 . . ا من هنا برزت مشكلة البحث الحالي من خلال ملاحظة الباحث لبعض الاتجاهات السلبية التي يبديها بعض المعلمين والمعلمات نحو طلاب صعوبات التعلم في مدارس التعليم العام.

أسئلة الدراسـة: السؤال الأول: ما اتجاهات كل من المعلمين والمعلمات بمنطقة الجهراء التعليمية نحو طلبة صعوبات التعلم بدولة الكويت ؟ 
السؤال الثاني: هل توجد فروق ذات دلالة إحصائية في اتجاهات المعلمين والمعلمات بمنطقة الجهراء التعليمية نحو طلبة صعوبات التعلم بدولة الكويت تعزى لمتغير الجنس؟

السؤال الثالث: هل توجد فروق ذات دلالة إحصائية في اتجاهات المعلمين والمعلمات بمنطقة الجهراء التعليمية نحو طلبة صعوبات التعلم بدولة الكويت تعزى لمتغير الخبرة التدريسية؟

السؤال الرابع : هل توجد فروق ذات دلالة إحصائية في اتجاهات المعلمين والمعلمات بمنطقة الجهراء التعليمية نحو طلبة صعوبات التعلم بدولة

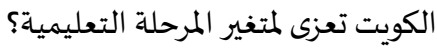

\section{الاطار النظري والدراسات السابقة:}

تعرف الحكومة الفيدرالية الأمريكية الصعوبات الخاصة في التعلم Specific Learning Difficulties بأنها وجود اضطراب في واحدة التهات أو أكثر

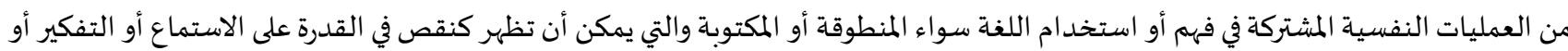

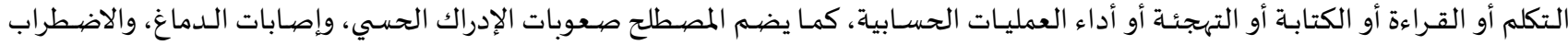

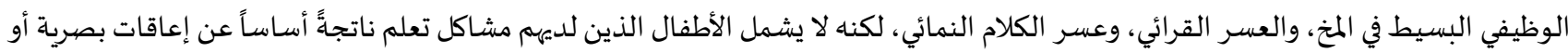

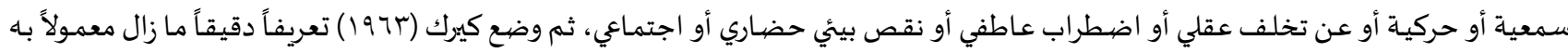

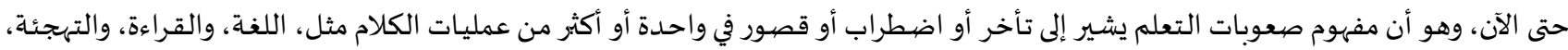

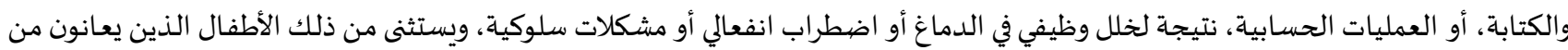

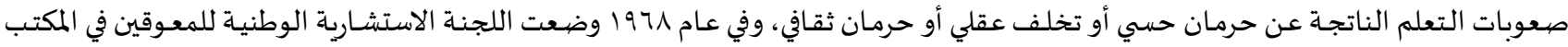

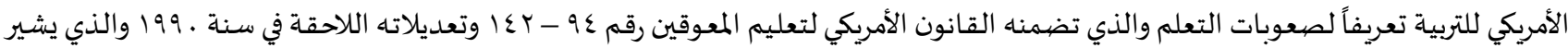

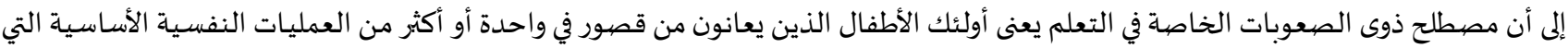

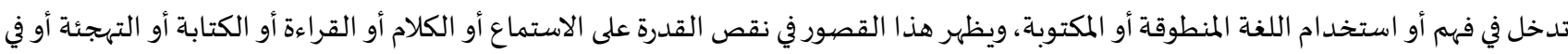

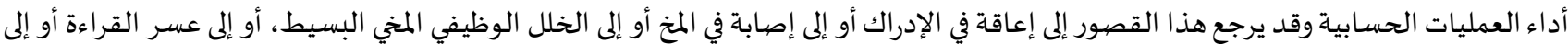

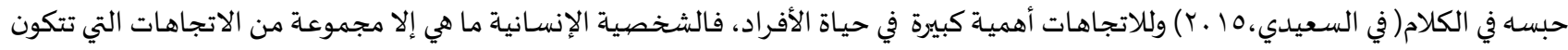

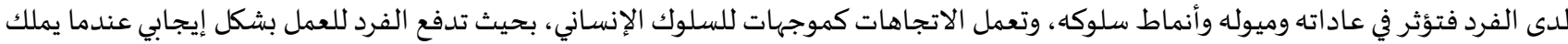

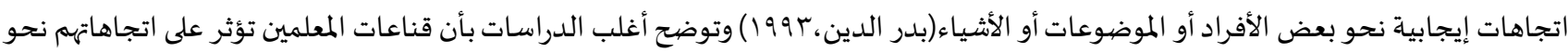

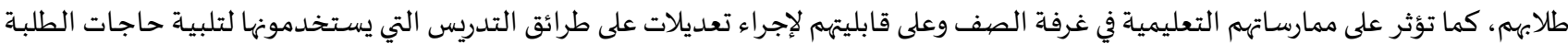

ذوي الخصائص المختلفة والخاصة ( الغازو:ع . . ب).

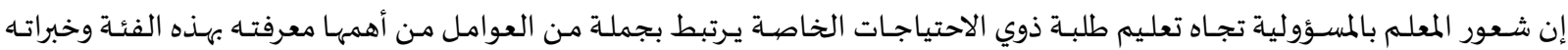

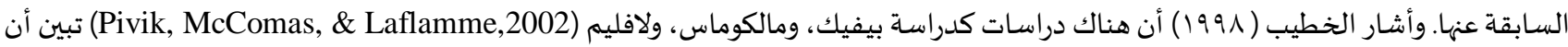

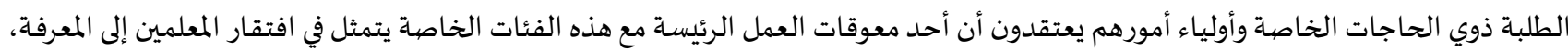
أو عدم تفهمهم ووضوح اتجاهاتهم، ولقد تعددت مفاهيم الاتجاهات فقد عرف كوبر وهاني (1999, Cooper and Haney) الاتجاه بأنه نزوع الفرد الفاد

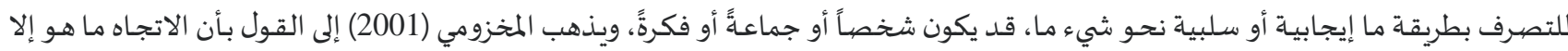
حالة فكرية عقلية أو موقف يكونه الإنسان إزاء شيء أو موضوع ما سواء أكان بالقبول أم بالرفض أم بالحياد، ويرى ( Andersen,2005) الاتجاه بأنه

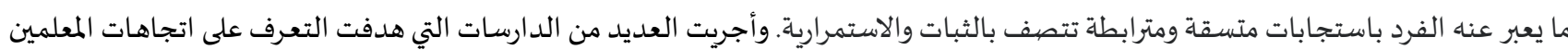

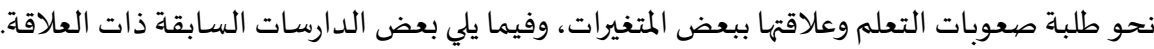

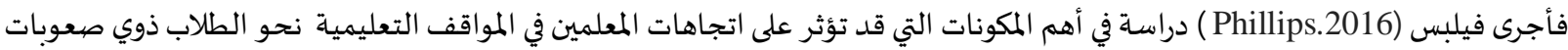

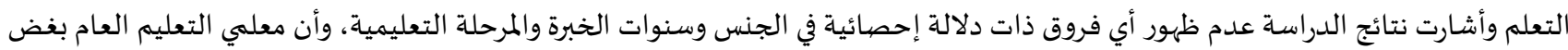

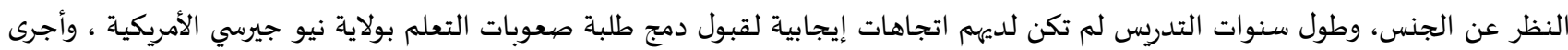

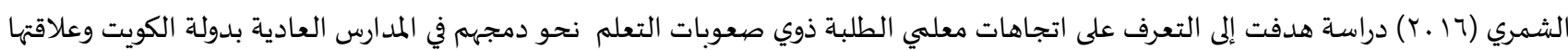

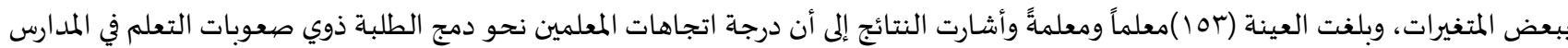

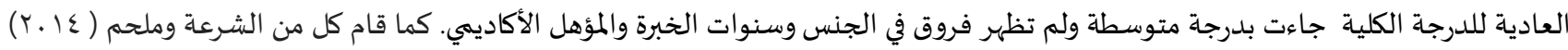

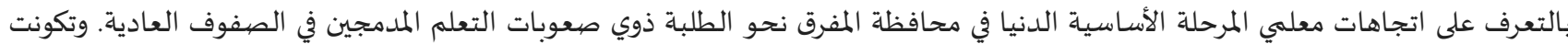

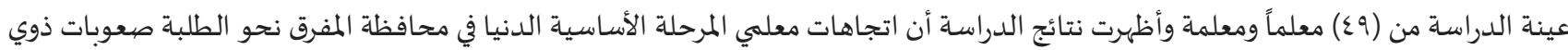


التعلم المدمجين في المد ارس العادية كانت مرتفعة، كما أنه لا توجد فروق ذات دلالة إحصائية في اتجاهات معلمي المرحلة الأسـاسية الدنيا نحو الطلبة

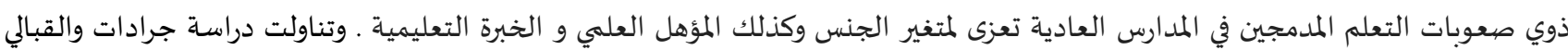
(r ا ـ التعرف على اتجاهات المعلمين نحو مشكلة صعوبات التعلم ، والتركيز على الجوانب الإيجابية بمحافظة الزرقاء في الأردن، وأظهرت النتائج بأن

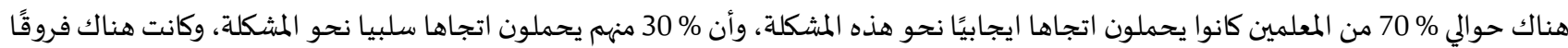
ذات دلالة إحصائية، تبعا للمتغيرات الثلاثة ( النوع، وسنوات الخبرة، والمستوى التعليمي) • وعمل كل من اديبولا وموني(2013 (Adebowale , Moye

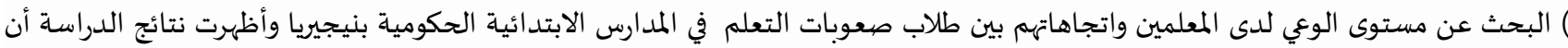

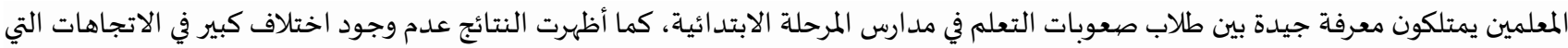

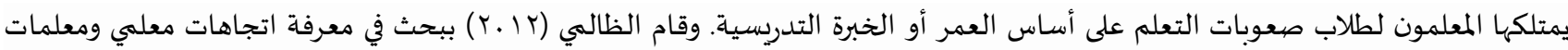

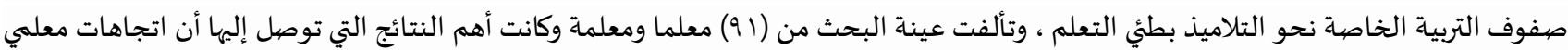
ومعلمات صفوف التربية الخاصية إيجابية ، وليس هناك فروق داله إحصائياء بين المعلمين والمعلمات نحو التلاميذ بطيء التعلم ، فاتجاهاتهم متقاربة

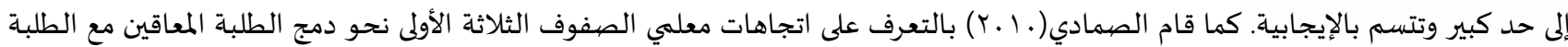

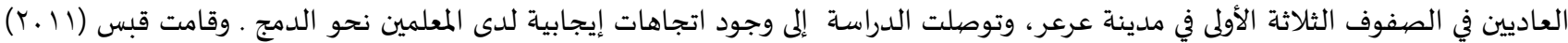

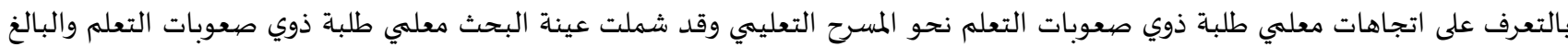

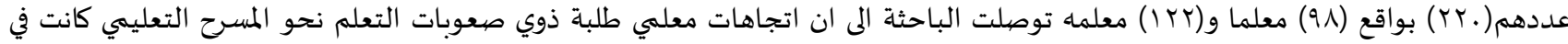
مستوى ايجابي مرتفع.

وبعد استعراض الدارسات السابقة يمكن للباحث تسليط الضوء على النى النقاط الآتية:

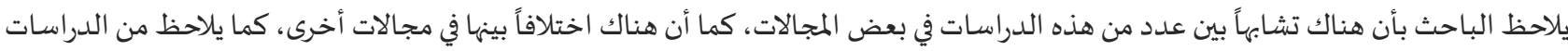

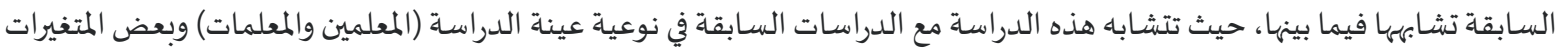

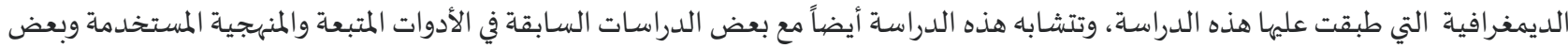

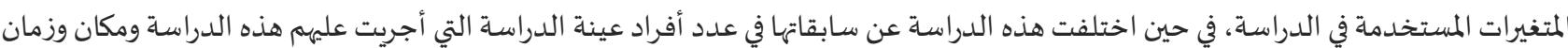

تطبيقها.

أهداف الدراسـة:

سعت هذه الدراسـة إلى تحقيق الأهداف الرئيسة الآتية: هدفت الدراسة الحالية إلى محاولة التعرف على اتجاهات كل من المعلمين والمعلمات بمنطقة الجهراء التعليمية نحو طلاب صعوبات التعلم في دولة الكويت.

هالتعرف على العلاقة بين اتجاهات المعلمين والمعلمات وبعض المتغيرات مثل الجنس والمؤهل العلمي والخبرة التدريسية. كما هدفت الدراسة إلى الوصول إلى توصيات خاصة بأهم الاتجاهات لدى المعلمين نحو طلاب صعوبات التعلم في دولة الكويت .

أهمية الدراسـة:

تنبثق أهمية هذه الدراسة في النقاط الآتية: إثراء معرفتنا حول موضوع اتجاهات المعلمين نحو طلابهم ذوي صعوبات التعلم من خلال دراسة مدى تأثير هذا الاتجاه على تدريسهم وتميزهم

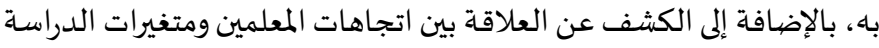
• تطوير أداة لقياس اتجاهات معلمي ومعلمات ذوي صعوبات التعلم مما قد يتيح الفرصاية لاستخد امها لاحقا إن معرفة اتجاهات المعلمين لطلابهم من ذوي صعوبات التعلم يساهم بشكل كبير إلى تطوير القدرات وزيادة الإبداع لدى المعلمين مما ينعكس بصورة ايجابية على الطلاب . توفير شكل من أشكال التغذية الراجعة للمد ارس وأولياء أمور طلبة صعوبات التعلم والمسؤولين بالميدان التربوي عن طبيعة هذه الاتجاهات والمفاهيم التي يمتلكها هؤلاء المعلمون.

مححددات الدارسـة: طبقت هذه الدارسة ضمن الحدود الآتية:

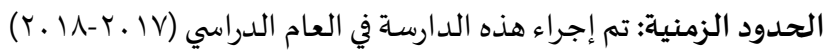


الحدود البشرية: اقتصرت هذه الدارسة على المعلمين والمعلمات المسجلين في منطقة الجهراء التعليمية في دولة الكويت. الحدود المكانية: اقتصرت هذه الدارسة على معرفة اتجاهات المعلمين والمعلمات المقيدين في منطقة الجهراء التعليمية في دولة الكويت

مصرطلحات الدارسـة:

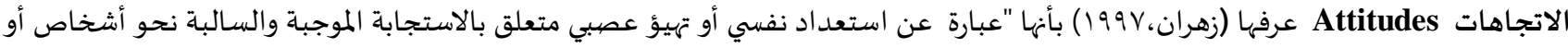

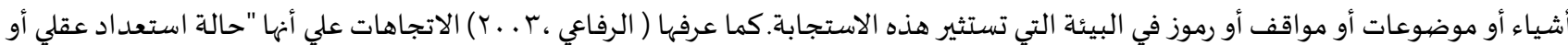
عصبي نُظِّمت عن طريق الخبرات الشخصية تعمل على توجياه استجابات الفرد لكل تلك الأشياء والمواقف التي تتعلق بهذا الاستعداد". ويعرف الباحث الاتجاه نحو طلبة صعوبات التعلم بأهها الدرجة التي يحصل عليها المعلمون والمعلمات على أداة الدراسة المتمثلة بمقياس اتجاتهاتيات المعلمين نحو طلبة صعوبات التعلم والذي أعده الباحث.

منهج الدارسة: لتحقيق أهداف الدارسة، والمتمثلة في الإجابة عن تساؤلاتها، تم استخدام المنهج الوصفي ، باعتباره المنهج الانسب لطبيعة الدارسة الحالية وأهدافها، وذلك من خلال جمع البيانات باستخدام الاستبانة التي تم إعدادها لهنه الهنه الغاية. مجتمع الدارسة: تكون مجتمع الدارسة من جميع معلمي ومعلمات منطقة الجهراء التعليمية نحو طلبة صعوبات التعلم في دولة الكويت للعام الدراسي

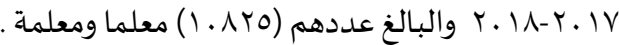
عينة الدارسة: تكونت عينة الدارسة من (وبا7) معلما ومعلمة تم اختيارهم بطريقة العينة العشوائية وهي تمثل ما نسبته (ᄉ\%) من مجتمع الدراسة وتعتبر ذسبة عالية جدا حسب معادلة (ريتشارد جيجر الاحصائية) والجدول (1) الآتي يوضح خصائص عينة الدارسة. جدول(1) خصائص العينة الديمغرافية

\begin{tabular}{|c|c|c|c|}
\hline النسبة المئوية & العدد & & \\
\hline$\% \varepsilon \varepsilon, \Lambda \Gamma$ & rAY & معلم & \multirow{3}{*}{ الجنس } \\
\hline$\% 00$, IV & $r \varepsilon V$ & معلمه & \\
\hline$\% 1 \ldots,$. & $7 r 9$ & المجموع & \\
\hline$\% Y \varepsilon, r Y$ & lor & أقل من خمس سنوات & \multirow[t]{4}{*}{ الخبرة التعليمية } \\
\hline$\% Y Y, . r$ & iv. & من (0 ـ ـ 1 ) سنوات & \\
\hline$\% \varepsilon \wedge, 70$ & $r .7$ & أكثر من عشر سنوات & \\
\hline$\% 1, \ldots, .$. & $7 r 9$ & المجموع & \\
\hline$\% \vee 7,90$ & $\varepsilon \wedge \varepsilon$ & جامعي & \multirow[t]{3}{*}{ لشهادة التعليمية } \\
\hline$\%$ \%r,.० & $1 \leqslant 0$ & دراسات عليا & \\
\hline$\% 1, \ldots, \ldots$ & $7 r 9$ & المجموع & \\
\hline$\% r q, \varepsilon r$ & $r \varepsilon \lambda$ & المرحلة الأبتدائية & \multirow[t]{4}{*}{ المرحلة التعليمية } \\
\hline$\% \varepsilon 0,1 r$ & rio & المرحلة المتوسطة & \\
\hline$\% 10, r 7$ & 97 & المرحلة الثانوية & \\
\hline$\% 1, \ldots,$. & $7 r 9$ & 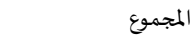 & \\
\hline
\end{tabular}

وبالرجوع إلى الجدول السابق نجد أن المعلمات يمثلن أعلى نسبة من المعلمين حيث كان هناك(Y (YV) معلمة بنسبة (Y) (\%) مقارنة مع المعلمين

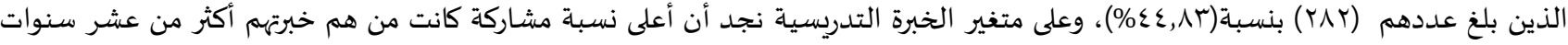

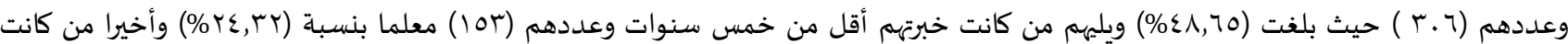

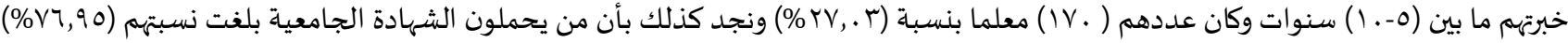

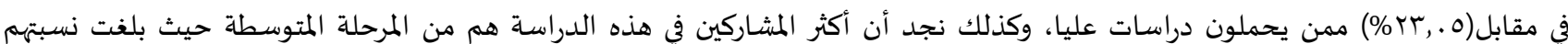

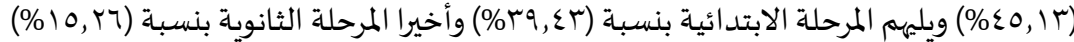
أداة الدارسـة:

للتعرف على اتجاهات معلمي ومعلمات نحو طلبة صعوبات التعلم في دولة الكويت من وجهة نظرهم وبعد الاطلاع على الأدب التربوي الدراسات

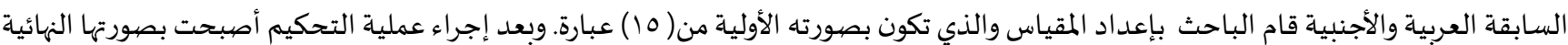

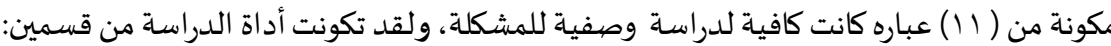


القسم الأول: ويتكون من معلومات عامة عن عينة الدارسة مثل الجنس، والخبرة التدريبية والمرحلة التعليمية.

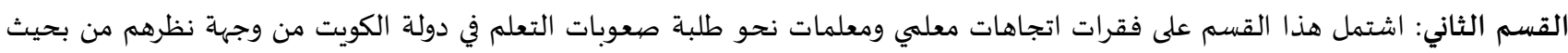

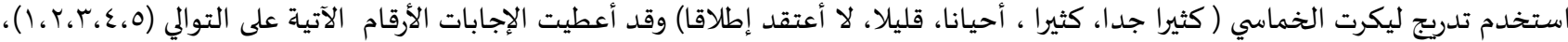

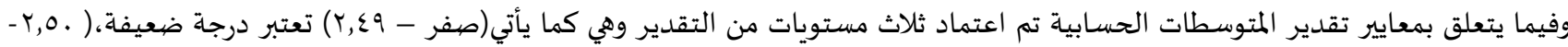

(r, 19

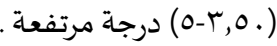

صبدق أداة البحث: للتحقق من صدق أداة الدارسة، فقد تم عرضها على مجموعة من المحكمين من ذوي الخبرة والاختصياص لمعرفة رأههم حول مدى صلاحية الفقرات

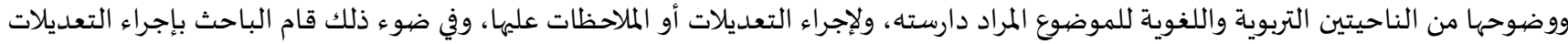
المقترحة من قبل المحكمين، والتي تضمنت استبعاد عدة فقرات. وتعديل البعض الآخر. وبالتالي أصبحت أداة الدارسة مكونة بصهورتها النهائية من(11) ولئه

ثبات أداة الدارسـة: تم التحقق من ثبات أداة الدارسة وذلك باستخراج معامل الاتساق الداخلي حسب معادلة ( cronbach's Alpha) حيث بلغت قيمة الثبات لأداة الدارسة(Y ع V, •) وبذلك تتمتع الأداة بدرجة مناسبة من الثبات لإجراء الدارسة الحالية .

التحليل الإحصيائي: استخدم الباحث عددا من الأساليب الإحصائية لتحليل البيانات والمعلومات التي تم جمعها من عينة الدارسة ، لمعرفة اتجاهات المعلمين

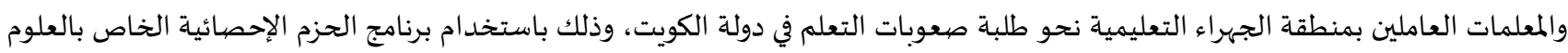

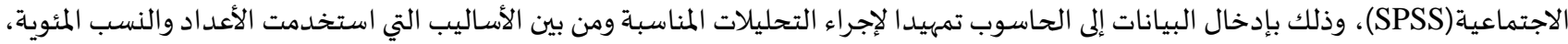
والمتوسطات الحسابية والانحرافات المعيارية، ولفحص الفروق استخدام Independent-t-test ، واختبار تحليل التباين الأحادي كما استخدمت معادلة) analysis of varianc

عرض نتائج الدارسة ومناقشتها: السؤال الأول للدارسة: ماهي اتجاهات كل من المعلمين والمعلمات بمنطقة الجهراء التعليمية نحو طلبة صعية صعوبات التعلم في دولة الكويت ؟ للإجابة عن هذا السؤال تم استخراج المتوسطات الحسابية والانحرافات المعيارية بشكل عام ولكافة فقرات أداة الدارسة وترتيها حسب أهميتها والجدول الآتي يوضح النتائج:

جدول (r) : المتوسطات الحسابية والانحرافات المعيارية لكافة عبارات أداة الدارسة

\begin{tabular}{|c|c|c|c|c|c|}
\hline الاستجابة & الانحراف المعياري & المتوسط الحسابي & 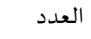 & 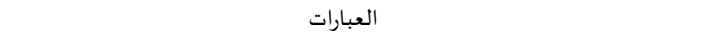 & \\
\hline 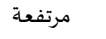 &., 90 & r, & $7 r 9$ & التربية الحديثة تدعو إلي استثمار أقصى قدرات طلاب صعوبات التعلم . & 1 \\
\hline 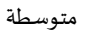 & $\cdot, \mathrm{TV}$ & $r, 00$ & $7 r 9$ & أعتقد أن بعض طلبة صعوبات التعلم يستطيع مواصلة الدراسة وتحقيق النجاح. & r \\
\hline 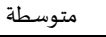 & $\cdot, 79$ & $r, . q$ & $7 r 9$ & أساعد طلبة صعوبات التعلم في التعبير عن ما يشعرون به بحرية. & r \\
\hline 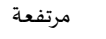 & $\cdot, 90$ & $r, v i$ & $7 r 9$ & مشاعر طلبة صعوبات التعلم لا تختلف عن مشاعر اقرانهم الآخرين. & $\varepsilon$ \\
\hline 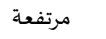 & $\cdot, 1 \varepsilon$ & r,v^ & $7 r 9$ & الايمان بمبدأ الفروق الفردية أحد مقومات المعلم الناجح. & ० \\
\hline 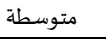 & $\cdot, 94$ & $r, \varepsilon_{0}$ & $7 r 9$ & يتميز طلبة صعوبات التعلم بالاتكالية وتدني دافعيتهم للتعلم. & 7 \\
\hline 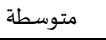 & $\cdot, \Lambda T$ & $r, .7$ & $7 r 9$ & طلبة صعوبات التعلم قليلو الاهتمام وضعاف الميول بشكل عام & $\mathrm{v}$ \\
\hline 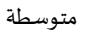 & $\cdot, 17$ & r,人 & $7 r 9$ & معرفتي في البرامج والأساليب التربوية الخاصة لطلاب صعوبات التعلم قليلة & $\wedge$ \\
\hline 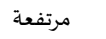 & $\cdot, \mathrm{VI}$ & $\varepsilon, .0$ & $7 r q$ & يتطلب العمل مع طلاب صعوبات التعلم جهودا مضاعفة مقارنة بأقرانهم العاديين & 9 \\
\hline ضيعيفة & $\cdot, \wedge$ & $r, . \wedge$ & $7 r 9$ & من الصعب مشاركة طلبة صعوبات التعلم في الأنشطة المدرسية & 1. \\
\hline 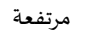 & $\cdot, \mathrm{V}$ & $\varepsilon, \cdot r$ & $7 r 9$ & أعتقد أن هناك حاجة ضرورية للمزيد من التوعية الاعلامية تجاه طلبة صعوبات التعلم & 11 \\
\hline 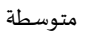 & $\cdot, 77$ & $r, r$. & $7 r q$ & المجموع الكلي للمعدل & \\
\hline
\end{tabular}


يتضح من الجدول(r) أن اتجاهات المعلمين والمعلمات في منطقة الجهراء التعليمية نحو طلبة صعوبات التعلم بدولة الكويت جاءت بشكل عام بدرجة

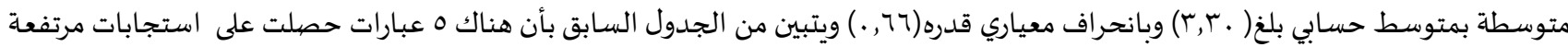

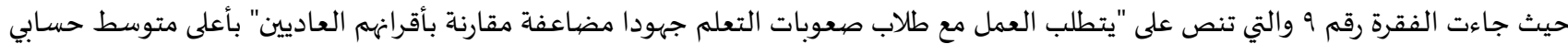

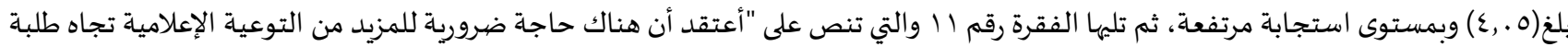
صعوبات التعلم" بمتوسط حسابي بلغ(ץ . ,ع) بمستوى استجابة مرتفعة، ثم تلهها الفقرة رقم ا والتي تنص على "أن التربية الحديثة تدعو إلى استثمار

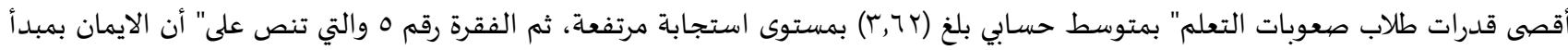
الفروق الفردية أحد مقومات المعلم الناجح" ، وأخيرا الفقرة رقم ع والتي تنص على " مشاعر طلبة صعوبات التعلم لا تختلف عن مشاعر أقرانهم

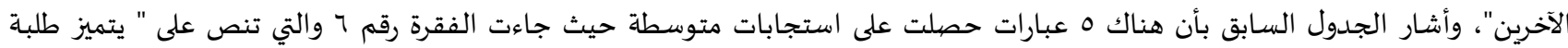

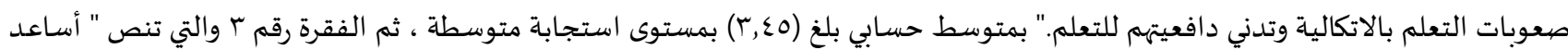

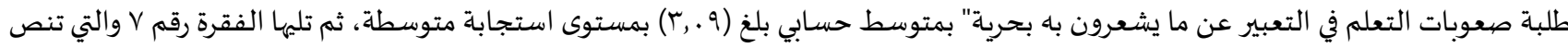

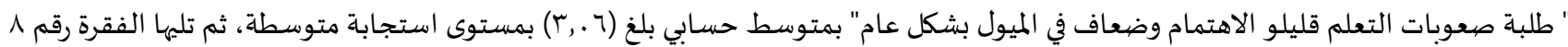

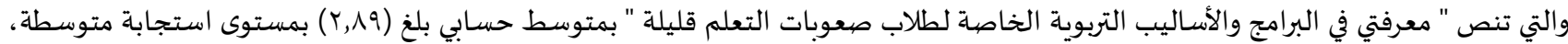
وأخيرا الفقرة رقم r والتي تنص " أعتقد أن بعض طلبة صعوبات التعلم يستطيع مواصلة الدراسة وتحقيق النجاح " بمتوسط حسابي بلغ (r,00) بمستوى استجابة متوسطة، وأشـار الجدول السابق أن هناك عبارة واحدة فقط حصلت مستوى ضعيف من الاستجابة وهي الفقرة رقم •ا التي تنص

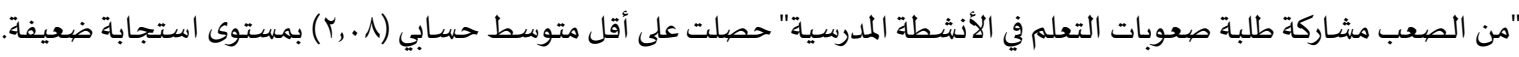
السؤال الثاني: هل توجد فروق ذات دلالة إحصائية في اتجاهات المعلمين والمعلمات بمنطقة الجهراء التعليمية نحو طلبة صعوبات التعلم بدولة الكويت تعزى لمتغير الجنس؟ للإجابة عن هذا السؤال وتم استخدام اختبار (T-Test) لمعرفة الفرق بين متوسط درجات المعلمين والمعلمات الجدول رقم (r) يوضح نتائج التي تم توصل إليها في هذا المجال جدول ( (T) المتوسطات والانحرافات المعيارية وقيمة (T) للفرق بين المعلمين والمعلمات

\begin{tabular}{|c|c|c|c|c|c|c|}
\hline مستوى الدلالة & قيمة T T & درجة الحرية & الانحراف المعياري & المتوسط الحسابي & العينة & الجنس \\
\hline \multirow[t]{2}{*}{., 9.0} & .,V.r & TTV & $r, \varepsilon \cdot r$ & $\varepsilon, 1$ lro $^{2}$ & TTV & معلم \\
\hline & & & $r, r .1$ & $\varepsilon, .91$ & $r . r$ & معلمة \\
\hline
\end{tabular}

يتضح من الجدول السابق عدم وجود فروق ذات دلالة احصائية تعزي لمتغير الجنس بين متوسطات درجات المعلمين والمعلمات علي المجموع العام

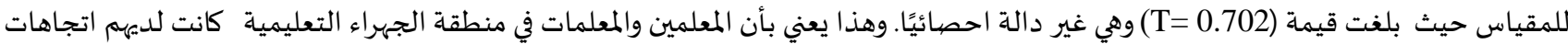
إيجابية نحو طلبة صعوبات التعلم بدولة الكويت. السؤال الثالث : هل توجد فروق ذات دلالة إحصائية في اتجاهات المعلمين والمعلمات بمنطقة الجهراء التعليمية نحو طلبة صعوبات التعلم بدولة

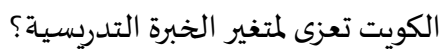
للإجابة عن هذا السؤال تم استخدام تحليل التبيان الأحادي لفحص الحبرد لتدردية دلالة الفروق بين متوسط درجات أفراد الدراسة على المقياس تبعا متغير الخبرة

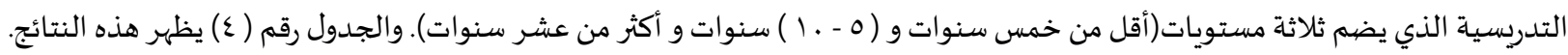

جدول (ع) نتائج تحليل التبيان الأحادي لفحص دلالة الفروق بين متوسط درجات أفراد الدراسة على أبعاد مقياس تبعا لمتغير الخبرة التعليمية

\begin{tabular}{|c|c|c|c|c|c|c|}
\hline مستوى & قيمة الدلالة & قيمة F & متوسط & درجة الحرية & مجموع & مصدر التباين \\
\hline \multirow[t]{3}{*}{ غير دالة } & \multirow[t]{3}{*}{$\cdot, \leq \leqslant 1$} & \multirow[t]{3}{*}{$\cdot, A Y$} & , rVq & r & -, vor & بين المجموعات \\
\hline & & &.,$\{71$ & $7 r o$ & rAN,Vor & داخل المجموعات \\
\hline & & & & 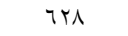 & $r \wedge 9,01$. & المجموع \\
\hline
\end{tabular}


يتضح من الجدول السابق عدم وجود فروق دالة إحصائيا في اتجاهات المعلمين والمعلمات بمنطقة الجهراء التعليمية نحو طلبة صعوبات التعلم في

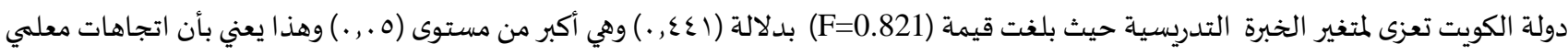

ومعلمات بمنطقة الجهراء التعليمية نحو طلبة صعوبات التعلم في دولة الكويت هي اتجاهات متقاربة وتتشابه فيما بينها.

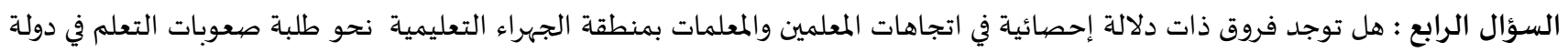

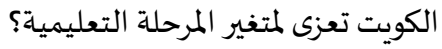

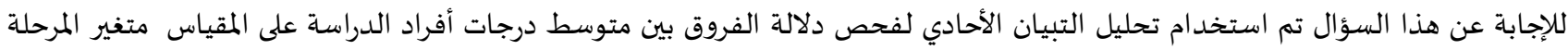

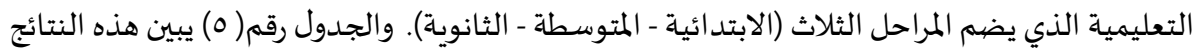

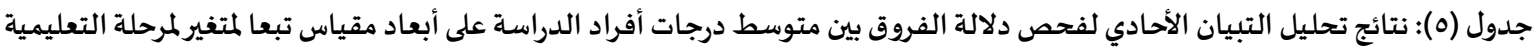

\begin{tabular}{|c|c|c|c|c|c|c|}
\hline مستوى الدلالة & قيمة الدلالة & قيمة F & متوسط & درجة الحرية & المربعات & مصدر التباين \\
\hline \multirow[t]{3}{*}{ غير دالة } & \multirow[t]{3}{*}{., 0.9} & \multirow[t]{3}{*}{., $7 \vee 7$} & . TIY & $r$ & . & بين المجموعات \\
\hline & & &.,$\{71$ & $7 T_{7}$ & $r \wedge \Lambda, \Lambda \Lambda \neg$ & داخل المجمموعات \\
\hline & & & & $7 Y \Lambda$ & $r \wedge 9,01$. & المجموع \\
\hline
\end{tabular}

يتضح من الجدول السابق عدم وجود فروق دالة إحصائيا في اتجاهات معلمي ومعلمات منطقة الجهراء التعليمية نحو طلبة صعوبات التعلم في دولة

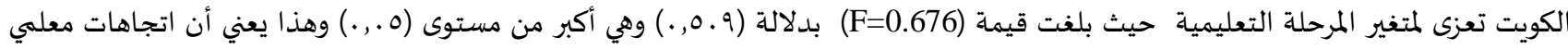
ومعلمات منطقة الجهراء التعليمية نحو طلبة صعوبات التعلم بدولة الكويت هي اتجاهات متقابِة مع اختلاف جميع المراحل التعليمية في دولة الكويت .

\section{مناقشسة نتائج الدارسـة}

كشفت نتائج الد ارسة الحالية عن وجود اتجاهات بدرجة متوسطة وقد تكون حيادية في اتجاهات المعلمين والمعلمات نحو طلبة صعوبات

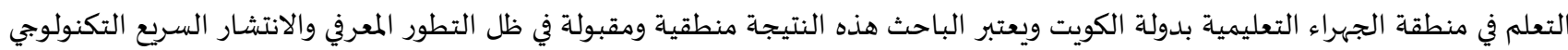
والذي أدي لتكوين اتجاهات جيدة لدى كل من المعلمين والمعلمات وإدراكهم من خلال العمل مع ذوي الاحتياجات الخاصة في المدرسة والذين منهم طلبة

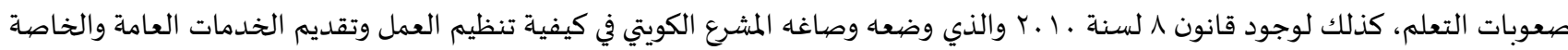
للأفراد ذوي الاحتياجات الخاصة، والذي ألزم الحكومة بتطبيق مواد هذا القانون في كل مؤسسات الدولة بما فهها وزارة التربية والتي قامت من خلال

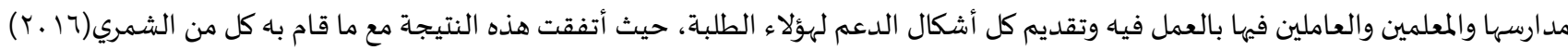

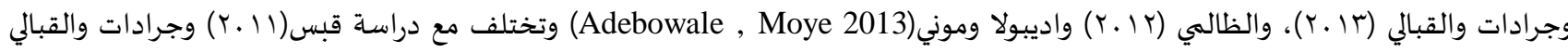

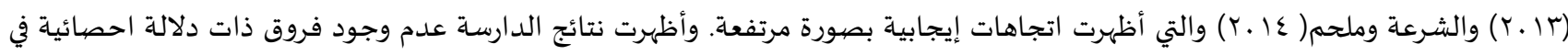

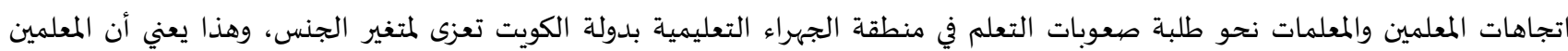

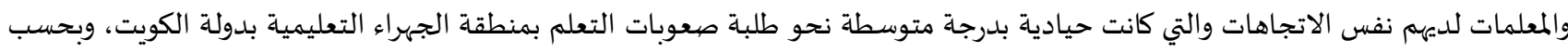

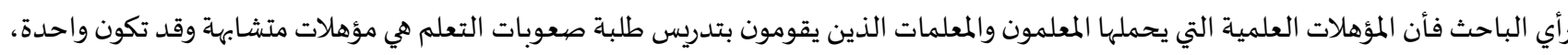

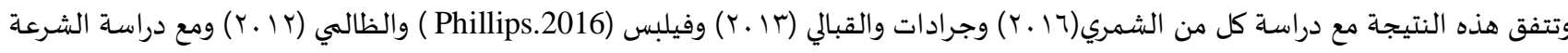

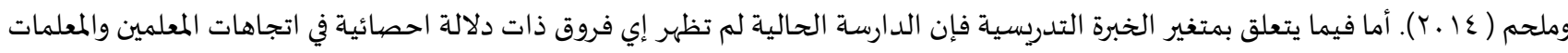

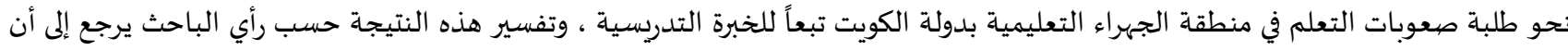

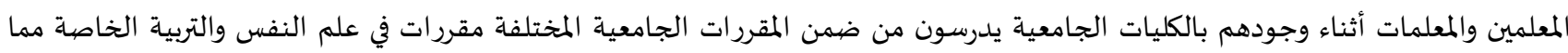

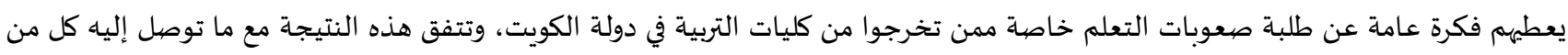

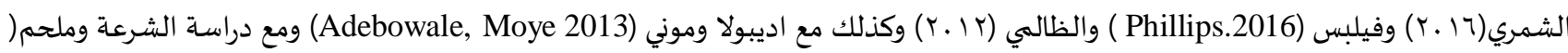

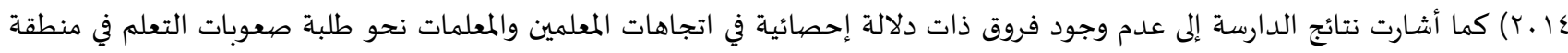

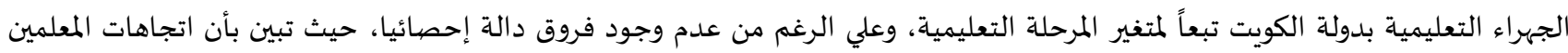

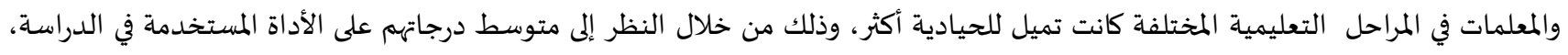

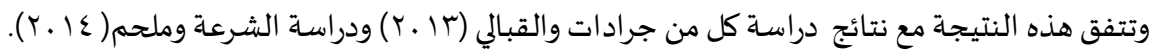


يمكن استخلاص بعض التوصيات بناءً على النتائج التي تم التوصل إلهها في هذه الدراسة من خلال النقاط التالية: أصبحت المعرفة بمستوى الاتجاهات في مجال تدريس طلبة صعوبات التعلم من الأساسيات التي يجب توافرها لدى المعلمين والمعلمات في المدارس الحكومية بدولة الكويت. العمل على تعزيز رفع مستوى الاتجاهات الايجابية عند المعلمين والمعلمات من خلال إقامة ورش العمل والدورات التدريبية والملتقيات الثقافية التي تهتم بتعريف صعوبات التعلم. التفعيل بتطبيق القوانين والتشريعات التي أقرت من قبل الدولة والتي تدعو وتحرص على التعريف بالأفراد من ذوي الاحتياجات الخاصية وخاصة ذوي الاعاقات التعليمية ومنها طلبة صعوبات التعلم من خلال ترسيخ السبل والوسائل الكفيلة بالمحافظة على هؤلاء الطلبة.

ا. الخطيب، جمال،(1991) مستوى معرفة معلمي الصفوف العادية بالصعوبات التعلمية وأثر برنامج لتطويره في القاعات التدريسية لهؤلاء المعلمين ورقة مقدمة إلى المؤتمر الدولي الأول لصعوبات التعلم الرياض- المملكة العربية السعودية المباية

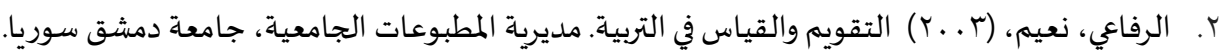
r. السعيدي، أحمد، العجمي. محمد، العنزي. سلامه، (0 ـr) الفروق في مركز الضبط وصعوبات المهارات الاجتماعية لدى التلاميذ ذوي صعوبات

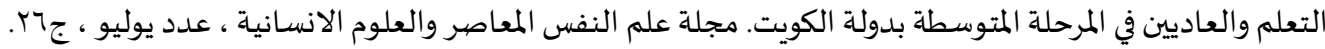

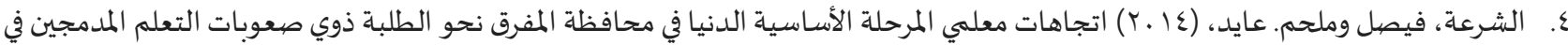

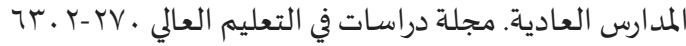
0. الشمري، مساعد، (7) •ب) اتجاهات معلمي الطلبة ذوي صعوبات التعلم نحو دمجهم في المدارس العادية بدولة الكويت وعلاقتها ببعض المتغيرات: رسالة ماجستير غير منشورة، الجامعة الأردنية. عمان .الأردن

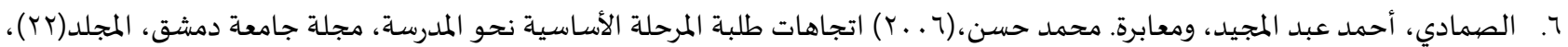

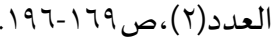

V. الصمادي، علي محمد (.1. (Y) اتجاهات المعلمين حول دمج الطلبة المعاقين في الصفوف الثلاثة الأولى مع الطلبة العاديين في محافظة

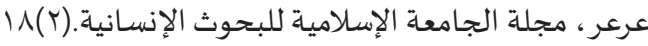

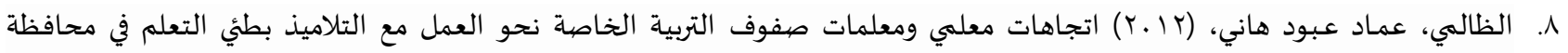

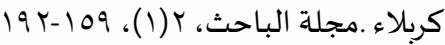
9 . الغازو، أحمد وآخرون، (ع . . . †) التعرف على اتجاهات الطلاب في الكليات التي تزود المدارس بالمعلمين نحو الأشخاص ذوي الاحتياجات الخاصة في الاردن والامارات العربية المتحدة: رسالة دكتور اه غير منشورة، كلية الدراسات العليا : الجامعة الاردنية، عمان ـ الاردن

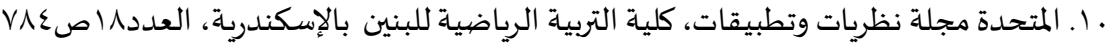

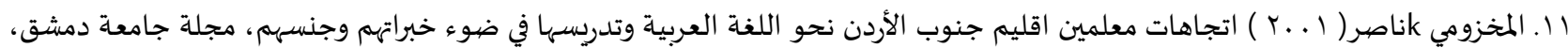

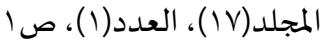
r ا. بدر الدين، طارق محمد،(ب991) دراسة مقارنة للاتجاهات نحو التربية الرياضية بين طالبات المدارس الثانوية بمحافظة الإسكندربة بمصر والمنطقة الشرقية بدولة الامارات العربية

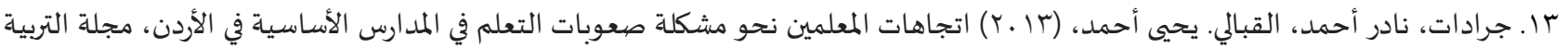

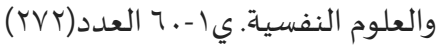

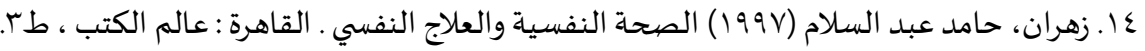

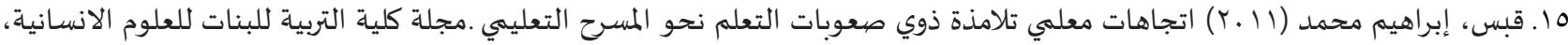
$1 \% 0-1.9 ،(9) 1$ 
[1] Adebowale, O. F., \& Moye, G. P., Teachers' Knowledge of, and Attitude towards Learning Disabilities. Online educational Journal, (2013)

[2] Andersen, M. B., Sport Psychology in Practice,Champaign, IL: Human Kinetics,(2005), https://doi.org/10.1002/9781444303650.ch12

[3] Cooper, J. \& Haney, J., Classroom Teaching Skills, (6thed). Houghton Mifflin, USA, (1999), p.64

[4] Phillips, R.R., Inclusion and Specific Learning Disabilities: General Education Teacher's Attitude. ProQuest LLC, (2016).

[5] Pivik, J., McComas, J., \& Laflamme, M., Barriers and facilitators to inclusive education. Exceptional Children, 69(1)(2002),97-107, https://doi.org/10.1177/001440290206900107 


\title{
The Level of Identifying the Directions of the Public Education Tutors on Students with Learning Disabilities in View of the Variances in the State of Kuwait
}

\author{
Ahmad Mohsen Al Saeedi \\ Associate Professor - Special education department - College of basic education \\ ahmed_alsadiey@yahoo.com
}

\begin{abstract}
The study focused on identifying the directions of the public education tutors on students with learning disabilities in view of the variances in the state of Kuwait, in addition to identification of the differences in these directions in relevance to the variances in (gender, tuition experience, and educational stage) for (629) tutors "female and males" and employees within the educational sector in Al Jahra educational region in the state of Kuwait. The results concluded that the directions of the tutors "female and males" within Al Jahra educational region in relevance to the identification of the directions of learning in the state of Kuwait are generally average, as well, the results concluded that no differences exist in relevance to the directions for the tutors "female and males" within the Al Jahra educational region, pertaining to the variances in (gender, tuition experience, and educational stage)
\end{abstract}

Keywords: directions - learning disabilities

\section{References:}

[1] Adebowale, O. F., \& Moye, G. P., Teachers' Knowledge of, and Attitude towards Learning Disabilities. Online educational Journal, (2013)

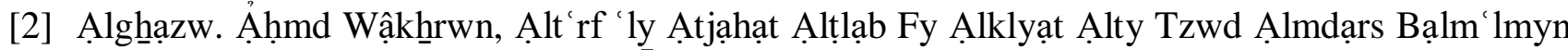
Nḥw Ạlạ̉shkhhạs Dḥwy Ạlạhtyạjạt Ạlkhạshh Fy Ạlạrdn Wạlạmạrạt Ạl'rbyh Ạlmthạdh: Rsạlẗ Dktwrạh Ghyr Mnsh̆hwrh, Klyẗ Ạldrạsạt Ạl'lyạ : Ạljạm'h Ạlạrdnyh, 'mạn . Ạlạrdn, (20004)

[3] Ạlkḥtyb. Jmạl, Mstwa M'rft M'lmy Ạlṣfwf Ạl ạdyh Bạlṣ 'wbạt Ạlt 'lmyh Wạ̉thِr Brnạmj Ltțwyrh

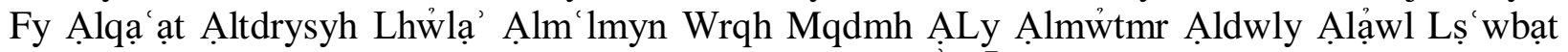
A.lt' 'lm A.̣ryạd- A. Almmlkh Ạl'rbyh A.̣ls wdyh, (1998)

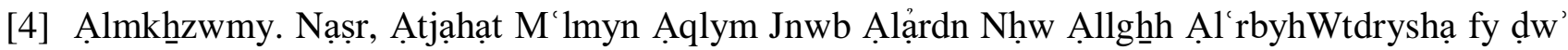
khbrạthm wjnshm, mjlï jạm ‘̈ dmsh̄q, 17(1)(2001), p.1

[5] Ạlmtḥdh. Mjlï Nẓryạt Wtṭbyqạt, Klyẗ Ạltrbyh Ạlryạḍh Llbnyn Bạlạskndryh, (18),p.784

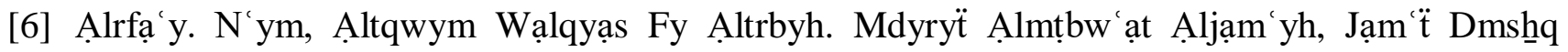
Swryạ, (2003)

[7] Ạls‘ydy. Ảhmmd, Ạl jumy. Mḥmd, Ạl'nzy. Slạmh, Ạlfrwq Fy Mrkz Ạlḍbṭ Wṣ̣ wbạt Ạlmhạrạt

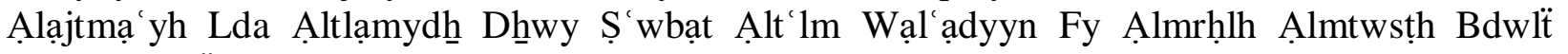
Ạlkwyt. Mjlit 'lm Ạlnfs Ạlm ‘̣̣șr Wạl' lwm Ạlạnsạnyh , 'dd Ywlyw , (2015), J26. 


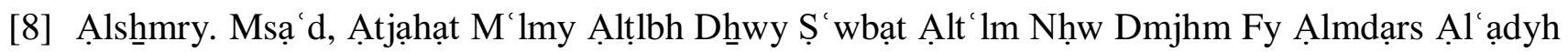

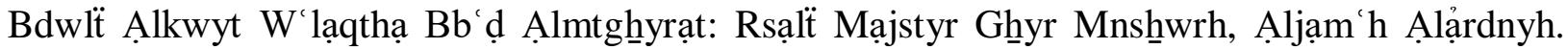
'mạn .Ạlậrdn, (2016)

[9] Ạlshrr'h. Fyṣl Wmlḥm. 'ạd, Ạtjạhạt M'lmy Ạlmrḥlh Ạlạ̉sạsyh Ạldnyạ Fy Mḥạfật Ạlmfrq Nḥw Ạlṭlbh Dhwwy Ș 'wbạt A.lt'lm A.lmdmjyn Fy Ạlmdạrs Ạl'ạdyh. Mjlt Drạsạt Fy Ạlt lym Ạl'ạly, (2014),270-6302

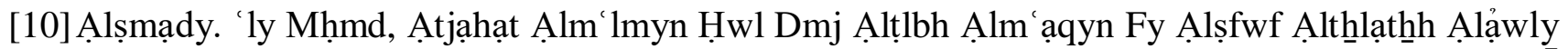

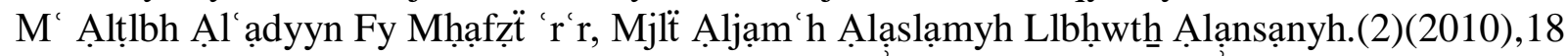

[11] Ạlṣmạdy. Ạhmo 'bd Ạlmjyd \& Wm’ạbrh. Mḥmd Ḥsn, Ạtjạhạt Ṭlbë Ạlmrḥlh Ạlạ̉sạsyh Nḥw Ạlmdrsh, Mjl̈̈ Jạm ‘̈ Dmshqq, 22(2)(2006),169-196.

[12] Ạlzạlmy. 'mạd 'bwd Hạny, Ạtjạhạt $M^{\prime}$ 'lmy Wm'lmạt Șfwf Ạltrbyh Ạlkḥạṣh Nḥw Ạl'ml M' Ạltlạmydh Bṭ̂yy Ạlt 'lm Fy Mḥạtạt Krblạ’ . Mjlï Ạlbạhṭ̆, 2(1)(2012),159-192

[13] Andersen, M. B., Sport Psychology in Practice,Champaign, IL: Human Kinetics,(2005), https://doi.org/10.1002/9781444303650.ch12

[14] Bdr Ạldyn. Ṭạrq Mḥmd, Drạsh Mqạrnh Llạtjạhạt Nḥw Ạltrbyh Ạlryạdyh Byn Ṭạlbạt Ạlmdạrs Ạlthạnwyh Bmhạafẓ̂t Ạlạskndryh Bmṣr Wạlmnțqh Ạlsḥrqyh Bdwlt Ạlạmạrạt Ạl'rbyh, (1993)

[15] Cooper, J. \& Haney, J., Classroom Teaching Skills, (6thed). Houghton Mifflin, USA, (1999), p.64

[16] Jrạdạt. Nạdr Ạ̉ḥmd \& Ạlqbạly. Yhyyy Ạ̉ḥmd, Ạtjạhạt Ạlm lmyn Nḥw Mshnklt Ș̣ wbạt Ạlt 'lm Fy Ạlmdạrs Ạlạ̉sạsyh Fy Ạlạ̉rdn, Mjlï Ạltrbyh Wạl l'lwm Ạlnfsyh, (272)(2013), Y1-60

[17]Phillips, R.R., Inclusion and Specific Learning Disabilities: General Education Teacher's Attitude. ProQuest LLC, (2016)

[18]Pivik, J., McComas, J., \& Laflamme, M., Barriers and facilitators to inclusive education. Exceptional Children, 69(1)(2002),97-107, https://doi.org/10.1177/001440290206900107

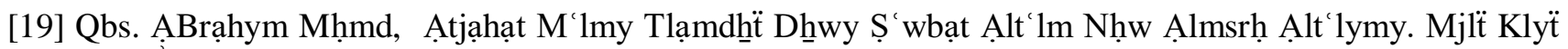
Ạltrbỷh Llbnạt Ll'lwm Ạlạnsạnyh, 8(9)(2011), 109-135

[20] Zhrạn. Ḥạmd 'bd Ạlslạm, Ạlș̣hh Ạlnfsyh Wạl'lạj Ạlnfsy, Ạlqạhrh:'ạlm Ạlktb, Ṭ3, (1997) 